\title{
Beam Wandering in the Atmosphere: The Effect of Partial Coherence
}

\author{
G.P. Berman, ${ }^{1 *}$ A.A. Chumak,,${ }^{1,2}$ and V.N. Gorshkov ${ }^{1,2}$ \\ ${ }^{1}$ Los Alamos National Laboratory, Theoretical Division, \\ Los Alamos, NM 87545 \\ ${ }^{2}$ Institute of Physics of the National Academy of Sciences \\ Pr. Nauki 46, Kiev-28, MSP 03028 Ukraine
}

October 25, 2018

LAUR-07-5341

\begin{abstract}
The effect of a random phase screen on laser beam wander in a turbulent atmosphere is studied theoretically. The method of photon distribution function is used to describe the photon kinetics of both weak and strong turbulence. By bringing together analytical and numerical calculations, we have obtained the variance of beam centroid deflections caused by scattering on turbulent eddies. It is shown that an artificial distortion of the initial coherence of the radiation can be used to decrease the wandering effect. The physical mechanism responsible for this reduction and applicability of our approach are discussed.
\end{abstract}

\footnotetext{
${ }^{*}$ Corresponding author: gpb@lanl.gov
} 


\section{Introduction}

When a beam of light propagates through the turbulent atmosphere of the Earth, it experiences random fluctuations in the refractive index. Fluctuations of the refractive index are due to turbulent eddies caused by stochastic variations of the temperature. The characteristic scales of the atmospheric inhomogeneities range from millimeters (the inner radius of the eddies, $l_{0}$ ) up to one hundred meters (the outer radius of the eddies, $L_{0}$ ). Those inhomogeneities which are large compared with the diameter of the beam tend to deflect the beam, whereas those inhomogeneities which are small compared with the beam diameter tend to broaden the beam but not deflect it significantly. As a result we can observe a broadened laser spot whose centroid randomly moves because of the motion of individual eddies. The average beam radius is determined by the overall scattering effect, i.e. by both the beam broadening and the centroid wandering averaged over a sufficiently long time.

Beam wandering, as well as the scintillation index, is an important characteristic of the radiation determining its utility for practical applications (for example, for purposes of uninterrupted laser tracking and pointing). Thus we will study here the possibility of controlling this effect by means of artificially decreasing the initial coherence of the radiation using a random phase screen. This screen introduces random (spatial and temporal) phase distortions into the wave front of the exiting beam. Therefore, after passing the phase screen, the initially coherent laser beam becomes partially coherent. Its coherence length, $l_{c}$, in the direction perpendicular to the direction of propagation becomes smaller than the diameter $D$ of the aperture. As a result the initial angular spread of the beam, that is due to diffraction, increases from $\lambda / D$ to $\lambda / l_{c}$, where $\lambda$ is the wavelength of the radiation. (See, for example, Refs. [1]-[3].) From the viewpoint of possible laser applications, the beam broadening is a negative factor that reduces the intensity of radiation field. At the same time, the wandering effect can become smaller just due to the broadening.

The above comments concern the case of not too long propagation paths when the diffraction broadening (which does really depend on the partial coherence) dominates over broadening caused by the atmospheric turbulence. But there is another important effect of the phase screen on the statistical properties of the radiation propagating in the atmosphere. It is shown in papers [4]-[8] that the decrease of the initial coherence may result in lowering 
the normalized variance of the intensity (i.e. the scintillation index) even in the case of strong turbulence. This effect takes place only for the case of a "slow" detector. The term "slow detector" means the detector has an integration time greater than the characteristic time of phase variation introduced by the phase screen. Since the suppression of the intensity fluctuations is of great practical importance, it is also interesting to study the behavior of the beam wandering (which is also expressed in terms of local fluctuations of the irradiance intensity) for the same experimental arrangement. Thus, in what follows the importance of a random phase screen for the case of strong turbulence will be elucidated.

To describe the effect of beam wandering, we will use here an approach based on the photon distribution function [6], 8].

\section{Theoretical description and calculations of the wander effect}

The position of beam centroid, $\mathbf{R}_{w}(z, t)$, is determined by the expression

$$
\mathbf{R}_{w}(z, t)=\frac{\int d \mathbf{r}_{\perp} \mathbf{r}_{\perp} I(\mathbf{r}, t)}{\int d \mathbf{r}_{\perp}\langle I(\mathbf{r}, t)\rangle},
$$

where $\langle\ldots\rangle$ means averaging over different realizations of the refractive index inhomogeneities and source fluctuations, $\mathbf{r}=\left\{\mathbf{r}_{\perp}, z\right\}, \mathbf{r}_{\perp}=\{x, y\}$, the $z$-axis is along the initial direction of the beam propagation; and the coordinate $\mathbf{r}=0$ corresponds to the center of the exit aperture.

Following Ref. [6] we express the intensity of photon flux $I(\mathbf{r}, t)$ in terms of the photon distribution function $f(\mathbf{r}, \mathbf{q}, t)$ as

$$
I(\mathbf{r}, t)=c \sum_{\mathbf{q}} \hbar \omega_{\mathbf{q}} f(\mathbf{r}, \mathbf{q}, t)
$$

where $c$ is the speed of light in a vacuum, $\omega_{q}=c q$,

$$
f(\mathbf{r}, \mathbf{q}, t)=\frac{1}{V} \sum_{\mathbf{k}} e^{-i \mathbf{k r}} b_{\mathbf{q}+\mathbf{k} / 2}^{+} b_{\mathbf{q}-\mathbf{k} / 2},
$$

$V=L_{x} L_{y} L_{z}$ is the normalizing volume, and $b_{\mathbf{q}}^{+}$and $b_{\mathbf{q}}$ are the creation and annihilation operators of photons with the wave-vector $\mathbf{q}$. The operator function $f(\mathbf{r}, \mathbf{q}, t)$ describes the photon density in $(\mathbf{r}, \mathbf{q})$ space at time $t$. For 
a detailed description of the photon field in a beam with radius $R_{b}$, it is sufficient to restrict the sum in Eq. (3) with some value $k_{0},|\mathbf{k}|<k_{0}$, where $R_{b}^{-1}<<k_{0}<<\lambda^{-1}$. In this case, the distribution function obeys the kinetic equation (see Ref. [6])

$$
\left\{\partial_{t}+\mathbf{c}_{\mathbf{q}} \partial_{\mathbf{r}}+\mathbf{F}(\mathbf{r}) \partial_{\mathbf{q}}\right\} f(\mathbf{r}, \mathbf{q}, t)=0
$$

where $\mathbf{c}_{\mathbf{q}}=\partial \omega_{q} / \partial \mathbf{q}, \mathbf{F}(\mathbf{r})=\omega_{0} \partial_{\mathbf{r}} n(\mathbf{r})$, and $n(\mathbf{r})$ is the fluctuating constituent of the atmospheric refractive index $(\langle n(\mathbf{r})\rangle=0,|n(\mathbf{r})|<<1) ; \omega_{0}=c q_{0}$ is the central frequency of laser radiation.

Using Eqs. (11) and (2), we can easily obtain the variance of $\mathbf{R}_{w}(z, t)$ if one knows the correlation function of the distribution function $\langle f f\rangle$. The analysis is very simple for the case of weak turbulence (or short propagation distance). In this case, it is convenient to use not directly Eq. (1), but a modified expression for it. The following consideration is in the spirit of Cook's approach [9] who has used the similarity between a parabolic equation describing paraxial optical beam and the Schrodinger equation. The application of the Ehrenfest's theorem has made it possible to develop an approximate method to study beam wandering effect in [9]. In contrast to Cook, we proceed not from Ehrenfest's theorem, but from the definition (11) and equation (4). A simple relationship between the beam centroid displacement and the refractive index fluctuations can be easily obtained within our formalism based on the kinetic equation Eq. (4)

$$
\left(\partial_{z}+\frac{1}{c} \partial_{t}\right)^{2} \mathbf{R}(z, t)=\int d \mathbf{r}_{\perp} \sum_{\mathbf{q}} \frac{\partial n(\mathbf{r})}{\partial \mathbf{r}_{\perp}} f(\mathbf{r}, \mathbf{q}, t)\left\langle\int d \mathbf{r}_{\perp} \sum_{\mathbf{q}} f(\mathbf{r}, \mathbf{q}, t)\right\rangle^{-1}
$$

for the case of a stationary beam. Here and in what follows, the paraxial approximation $\left(\left|\mathbf{q}-\mathbf{q}_{0}\right|<<q_{0}\right)$ is assumed throughout the beam trajectory.

In the lowest order with respect to fluctuating refractive index, the dependence of $f$ on $n(\mathbf{r})$ in (5) has to be neglected. Therefore, the variance of beam wandering is given by

$$
\begin{gathered}
\left\langle\mathbf{R}_{w}^{2}\right\rangle=\int_{0}^{z} \int_{0}^{z} d z_{1} d z_{2}\left(z-z_{1}\right)\left(z-z_{2}\right) \times \\
\int d \mathbf{r}_{\perp} d \mathbf{r}_{\perp}^{\prime} \sum_{\mathbf{q q}^{\prime}}\left\langle\frac{\partial n(\mathbf{r})}{\partial \mathbf{r}_{\perp}} \frac{\partial n\left(\mathbf{r}^{\prime}\right)}{\partial \mathbf{r}_{\perp}^{\prime}}\right\rangle\left\langle f(\mathbf{r}, \mathbf{q}, t) f\left(\mathbf{r}^{\prime}, \mathbf{q}^{\prime}, t\right)\right\rangle\left\langle\int d \mathbf{r}_{\perp} \sum_{\mathbf{q}} f(\mathbf{r}, \mathbf{q}, t)\right\rangle^{-2}
\end{gathered}
$$


where $\mathbf{r}=\left\{\mathbf{r}_{\perp}, z_{1}\right\}, \mathbf{r}^{\prime}=\left\{\mathbf{r}_{\perp}^{\prime}, z_{2}\right\}$, and $f(\mathbf{r}, \mathbf{q}, t)$ satisfies Eq. (4) with $\mathbf{F}=0$. The distribution function at time $t$ can be expressed via its value at the instant of photon exit from the source, $t_{0}$, as

$$
f(\mathbf{r}, \mathbf{q}, t)=f\left[\mathbf{r}-\mathbf{c}_{\mathbf{q}}\left(t-t_{0}\right), \mathbf{q}, t=t_{0}\right]=\left.\frac{1}{V} \sum_{\mathbf{k}} e^{-i \mathbf{k}\left[\mathbf{r}-\mathbf{c}_{\mathbf{q}}\left(t-t_{0}\right)\right]} b_{\mathbf{q}+\mathbf{k} / 2}^{+} b_{\mathbf{q}-\mathbf{k} / 2}\right|_{t=t_{0}}
$$

where $t-t_{0}=z / c$. In what follows we will put $t_{0}=0$ for simplicity.

Two independent averagings should be undertaken in the right-hand part of Eq. (6). These are averaging over the turbulence configurations, $\langle n n\rangle$, and averaging over the fluctuations of the source, including fluctuations introduced by the random phase screen, $\langle f f\rangle$. The first of these is determined by the known [10] expression

$$
\left\langle n(\mathbf{r}) n\left(\mathbf{r}^{\prime}\right)\right\rangle=\int d \mathbf{g} e^{-i \mathbf{g}\left(\mathbf{r}-\mathbf{r}^{\prime}\right)} \psi(\mathbf{g}),
$$

where the explicit term for $\psi$ is given by

$$
\psi(\mathbf{g})=0.033 C_{n}^{2} \frac{\exp \left[-\left(g l_{0} / 2 \pi\right)^{2}\right]}{\left[g^{2}+L_{0}^{-2}\right]^{11 / 6}} .
$$

Eq. (9) is referred to as the von Karman spectrum.

The other averaging accounting for the effect of a "slow" detector is given by [6]:

$$
\begin{gathered}
\left\langle f(\mathbf{r}, \mathbf{q}, t) f\left(\mathbf{r}^{\prime}, \mathbf{q}^{\prime}, t\right)\right\rangle=\left(\frac{2 \pi r_{1}^{2}}{V L_{x} L_{y}}\right)^{2} \delta_{q_{z}, q_{0}} \delta_{q_{z}^{\prime}, q_{0}}\left\langle b^{+} b^{+} b b\right\rangle \times \\
\sum_{\mathbf{k}_{\perp}, \mathbf{k}_{\perp}^{\prime}} e^{-i \mathbf{k}_{\perp}\left(\mathbf{r}_{\perp}-\mathbf{q}_{\perp} z_{1} / q_{0}\right)-i \mathbf{k}_{\perp}^{\prime}\left(\mathbf{r}_{\perp}^{\prime}-\mathbf{q}_{\perp}^{\prime} z_{2} / q_{0}\right)} e^{-\left(k_{\perp}^{2}+k_{\perp}^{\prime 2}\right) r_{0}^{2} / 8-\left(q_{\perp}^{2}+q_{\perp}^{\prime 2}\right) r_{1}^{2} / 2}
\end{gathered}
$$

where $b^{+}, b$ are the operators of the generated mode. The effect of the phase screen is represented in Eq. (10) by the parameter $r_{1}$, determined via the correlation length, $\lambda_{c}$, of phase variation due to the phase screen as

$$
r_{1}^{2}=\frac{r_{0}^{2}}{1+2 r_{0}^{2} \lambda_{c}^{-2}}
$$

In the absence of a phase screen, we may set formally $\lambda_{c}=\infty$. Then it follows from Eq. (11) that in this case $r_{1}=r_{0}$. For any finite value of $\lambda_{c}, r_{1}<r_{0}$ and, as follows from Eq. (10), the characteristic values of the 
transverse momenta of photons $q_{\perp}, q_{\perp}^{\prime}$ are increased. This means that the beam becomes more divergent after passing a phase screen .

With the known explicit terms (9) and (10), the calculation of $\left\langle\mathbf{R}_{w}^{2}\right\rangle$ reduces to many-fold integrations which can be performed straightforwardly. The result is given by

$$
\left\langle\mathbf{R}_{w}^{2}\right\rangle=0.066 \pi^{2} \Gamma\left(\frac{1}{6}\right) C_{n}^{2} z^{8 / 3}\left(q_{0} r_{1}\right)^{1 / 3} I_{1} .
$$

The dimensionless quantity $I_{1}$ is determined by the integral

$$
I_{1}=\int_{0}^{1} d x(x-1)^{2}\left[x^{2}+\left(r_{0}^{2} / 4+l_{0}^{\prime 2}\right) q_{0}^{2} r_{1}^{2} z^{-2}\right]^{-1 / 6}
$$

where $l_{o}^{\prime}=l_{0} /(2 \pi)$. It can be calculated numerically. In the limiting cases of short and long propagation distances it is given by the following analytical expressions:

$$
\begin{gathered}
I_{1} \approx \frac{1}{3}\left(\frac{z}{q_{0} r_{1}}\right)^{1 / 3}\left(l_{0}^{\prime 2}+r_{0}^{2} / 4\right)^{-1 / 6}, \text { when } q_{0}^{2} r_{1}^{2} z^{-2}\left(l_{0}^{\prime 2}+r_{0}^{2} / 4\right)>>1 \\
I_{1} \approx \frac{27}{40}, \text { when } q_{0}^{2} r_{1}^{2} z^{-2}\left(l_{0}^{\prime 2}+r_{0}^{2} / 4\right)<<1 .
\end{gathered}
$$

Usually $\left(r_{0}^{2} / 4\right)>>l_{0}^{\prime 2}$. Then, the above criteria mean that the diffraction broadening is smaller (upper case) and greater (lower case) than the initial beam radius. The upper case in Eqs. (14) results in $\left\langle\mathbf{R}_{w}^{2}\right\rangle=1.919 C_{n}^{2} z^{3}\left(2 r_{0}\right)^{-1 / 3}$, that coincides exactly with the classic formula presented in the reference [11]. (See Eq. (45) there.) As we see, there is no dependence of beam wandering on the phase screen when the propagation distance is very short. The result is evident for this limiting case in view of the fact that both the diffraction broadening and the broadening due to the atmosphere turbulence is much smaller than the initial radius of the beam. With the increase of propagation distance, $z$, or decrease of the initial coherence, the upper case in Eqs. (14) may transform to the lower case which corresponds to dominating diffraction broadening of the beam. Then the dependence $\left\langle\mathbf{R}_{w}^{2}\right\rangle \sim r_{1}^{1 / 3}$ will arise. As we see $\left\langle\mathbf{R}_{w}^{2}\right\rangle$ decreases with decreasing initial coherence. In this case the variance of the wander distance can be controlled by a suitable choice of the random phase screen.

The situation is much more complex when the turbulence is strong. The averaging is no longer decoupled in the manner shown in Eq. (66). An essential dependence of the distribution function on turbulence takes place here. 
Therefore the approach based on employing Eq. (5) is not advantageous. The simplest way for further analysis is to proceed from the initial definition of the wandering given by Eq. (1). The expression for the distribution function $f(\mathbf{r}, \mathbf{q}, t)$ is given by [6]

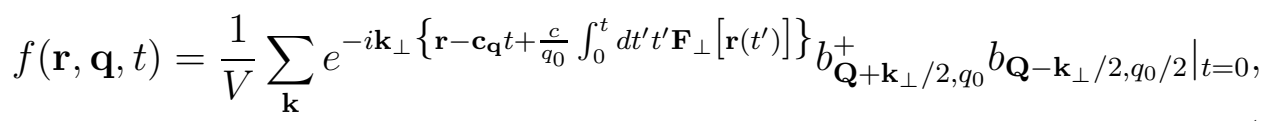

where $\mathbf{q}=\left\{\mathbf{q}_{\perp}, q_{0}\right\}, \mathbf{Q}=\mathbf{q}_{\perp}-\int_{0}^{t} d t^{\prime} \mathbf{F}_{\perp}\left[\mathbf{r}\left(t^{\prime}\right)\right]$, and $\mathbf{r}\left(t^{\prime}\right)$ is the trajectory of particle, which has the velocity $\mathbf{c}_{\mathbf{q}\left(t^{\prime}\right)}$ and is affected by the force $\mathbf{F}$. The initial conditions are given by $\mathbf{r}\left(t^{\prime}=t\right)=\mathbf{r}$ and $\mathbf{q}\left(t^{\prime}=t\right)=\mathbf{q}$.

Substituting Eq. (15) into the general expression

$$
\iint d \mathbf{r}_{\perp} d \mathbf{r}_{\perp}^{\prime} \mathbf{r}_{\perp} \mathbf{r}_{\perp}^{\prime}\left\langle I(\mathbf{r}, t) I\left(\mathbf{r}^{\prime}, t\right)\right\rangle,
$$

which determines the mean-square variation of the wander distance, and averaging over phase variations introduced by the random phase screen, we arrive at

$$
\begin{aligned}
& \left(\frac{2 \pi r_{1}^{2} c \hbar \omega}{V L_{x} L_{y}}\right)^{2}\left\langle b^{+} b^{+} b b\right\rangle \sum_{\mathbf{k}_{\perp}, \mathbf{q}_{\perp}} \sum_{\mathbf{k}_{\perp}^{\prime}, \mathbf{q}_{\perp}^{\prime}} \int d \mathbf{r}_{\perp} d \mathbf{r}_{\perp}^{\prime} \mathbf{r}_{\perp} \mathbf{r}_{\perp}^{\prime} e^{-i \mathbf{k}_{\perp}\left(\mathbf{r}-\mathbf{c}_{\mathbf{q}} t\right)-i \mathbf{k}_{\perp}^{\prime}\left(\mathbf{r}^{\prime}-\mathbf{c}_{\mathbf{q}^{\prime}} t\right)} \\
& \times e^{-\left(k_{\perp}^{2}+k_{\perp}^{\prime 2}\right) r_{0}^{2} / 8}\left\langle e^{-\left(Q^{2}+Q^{\prime 2}\right) r_{1}^{2} / 2-\left(i c / q_{0}\right) \int_{0}^{t} d t^{\prime} t^{\prime}\left[\mathbf{k}_{\perp} \mathbf{F}\left(\mathbf{r}\left(t^{\prime}\right)\right)+\mathbf{k}_{\perp}^{\prime} \mathbf{F}\left(\mathbf{r}^{\prime}\left(t^{\prime}\right)\right)\right]}\right\rangle
\end{aligned}
$$

where the last averaging is over random values of the refraction index. It is worth mentioning that momenta $\mathbf{Q}$ and $\mathbf{Q}^{\prime}$ depend linearly on $\mathbf{F}$. Therefore it is convenient to rewrite the quantity in the last angle bracket in a more convenient equivalent form as

$$
\begin{aligned}
& \langle\ldots\rangle=\frac{1}{\left(2 \pi r_{1}^{2}\right)^{2}} \iint d \mathbf{p} d \mathbf{p}^{\prime} e^{i\left(\mathbf{p q}+\mathbf{p}^{\prime} \mathbf{q}^{\prime}\right)-\left(p^{2}+{p^{\prime}}^{2}\right) /\left(2 r_{1}^{2}\right)} \\
& \times\left\langle e^{-i \int_{0}^{t} d t^{\prime}\left[\left(\frac{c}{q_{0}} \mathbf{k}_{\perp} t^{\prime}+\mathbf{p}\right) \mathbf{F}\left[\mathbf{r}\left(t^{\prime}\right)\right]+\left(\frac{c}{q_{0}} \mathbf{k}_{\perp}^{\prime} t^{\prime}+\mathbf{p}^{\prime}\right) \mathbf{F}\left[\mathbf{r}^{\prime}\left(t^{\prime}\right)\right]\right]}\right\rangle,
\end{aligned}
$$

where $\mathbf{p}$ and $\mathbf{p}^{\prime}$ are vectors perpendicular to the $z$-axis.

Thus, the problem is reduced to the calculation of manyiple integral. There is a 13-fold integration in Eq. (16). After substituting Eq. (17) into (16), the number of integrations increases to 17. Besides that, averaging over fluctuations of the refractive index introduces four additional integrations. 


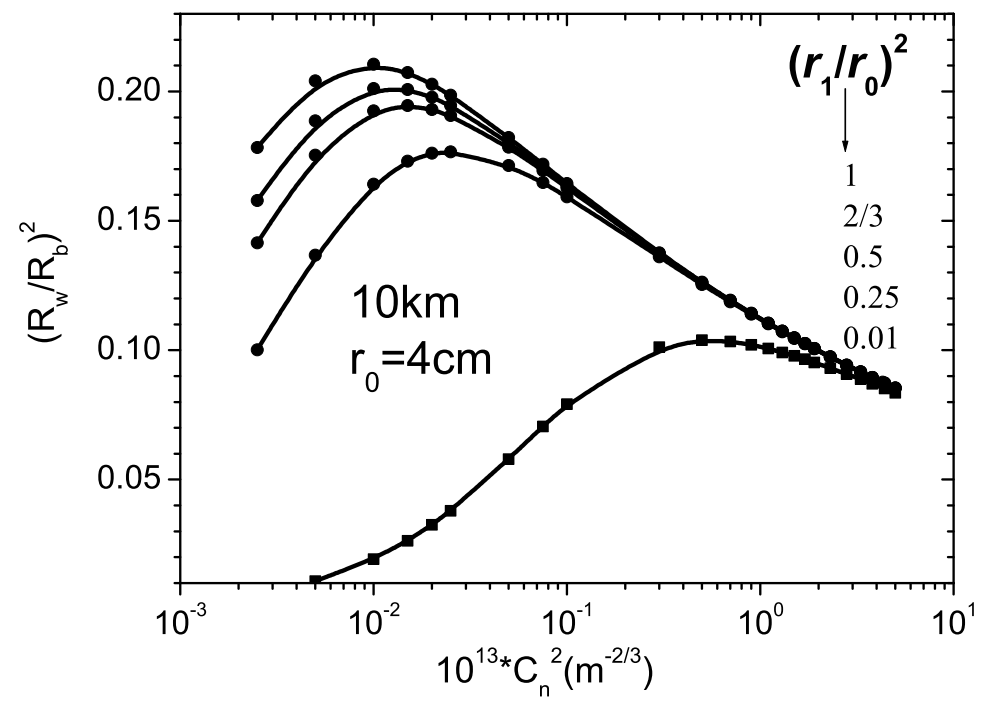

Figure 1: Dimensionless mean square wandering radius vs the turbulence strength $C_{n}^{2}$ for different values of the partial coherence determined by the ratio $r_{1}^{2} / r_{0}^{2}$. For all curves $q_{0}=10^{7} \mathrm{~m}^{-1}, l_{0}^{\prime}=10^{-3} \mathrm{~m}$. The symbols show the results of numerical calculations. For visual convenience, the solid lines connect symbols using B-spline approximations.

Finally, we have a 21-fold integral. We have performed most integrations analytically. The corresponding procedure is similar to that described in Ref. [6]. The rining, 5-fold integral, has been calculated numerically. Fig. 1 shows the results for fixed values of the aperture $\left(r_{0}=4\right)$ and propagation distance $(z=10 \mathrm{~km})$. We plot the dependence of the dimensionless quantity $\left\langle R_{w}^{2}\right\rangle / R_{b}^{2}$ on the turbulence strength. (It is the ratio $\left\langle R_{w}^{2}\right\rangle / R_{b}^{2}$ rather than merely $\left\langle R_{w}^{2}\right\rangle$ that is informative about the practical significance of the wandering.) The beam radius $R_{b}$ is given by the expression [6]

$$
R_{b}^{2}=\frac{r_{0}^{2}}{2}\left[1+\frac{4 z^{2}}{q_{0}^{2} r_{0}^{2} r_{1}^{2}}+\frac{8 z^{3} T}{r_{0}^{2}}\right],
$$

where $T=0.558 C_{n}^{2} l_{0}^{-1 / 3}$.

As we see in Fig. 1, there is still considerable beam wander even for very strong turbulence, i.e. for $C_{n}^{2}=5 * 10^{-13} m^{-2 / 3}$. (Usually, the value $C_{n}^{2}=$ 
$10^{-14} m^{-2 / 3}$ is considered to be a moderate turbulence level.) The four curves merge into a single curve when $C_{n}^{2} \rightarrow \infty$. This means that the wandering does not depend on the initial coherence in this case. So, the universal behavior of the wandering effect corresponds to the general concept that the atmosphere controls beam parameters for long-distance propagation or for the strong turbulence regime. At the same time, we see here the general tendency of the wander to decay with the increasing turbulence strength supports the reasonings of Fante [10]. He considered that when the turbulence is strong, the beam no longer wanders significantly, but rather breaks up into multiple beams.

In the opposite limiting case, $C_{n}^{2} \rightarrow 0$, the wander distance $R_{w}$ also tends to zero due to the obvious fact that the wandering is entirely caused by turbulence. From a formal point of view, there should be at least one maximum in the curve which connects the regions of weak and strong turbulence. The corresponding physical picture can be explained in terms of two competitive tendencies occurring when $C_{n}^{2}$ increases: (i) in the range of weak turbulence, where the beam radius is almost independent of the turbulence, the probability to meet sufficiently strong large-scale fluctuation of the refractive index, which deflects the beam as a whole, increases linearly with $C_{n}^{2}$, (ii) in the range of strong turbulence, there is considerable beam widening due to photon scattering on fluctuations of the refractive index $\left(R_{b}^{2} \sim C_{n}^{2}\right)$; therefore the previous possibility has a low probability. This explains the presence of the maxima in Fig. 1.

It is interesting to compare the results of the weak-turbulence theory given by Eq. (12) with those of more general approach based on the distribution function (15)). The results are shown in Fig. 2. As we see, both approaches give almost coinciding data for small values of $C_{n}^{2}$. When $C_{n}^{2}$ increases, the results of the weak-turbulence theory are overstated. A similar picture was observed in Ref. [12] where the weak-turbulence theory was tested by means of computer simulations.

Fig. 3 illustrates the dependence of beam wander on $C_{n}^{2}$ for a shorter distance $(5 \mathrm{~km})$ than in Fig. 1. The plots in Figs. 1 and 3 are very similar with the only difference that the maxima in Fig. 3 are displaced to the range of greater values of the turbulence strength $C_{n}^{2}$. This difference is quite evident. Namely, initially the overall effect of the turbulence increases with the increase of both the value of $C_{n}^{2}$ and the distance $z$. Therefore, the decrease of one of the factors can be compensated by the increase of the other one, thus providing almost the same effect of the turbulent atmosphere. 


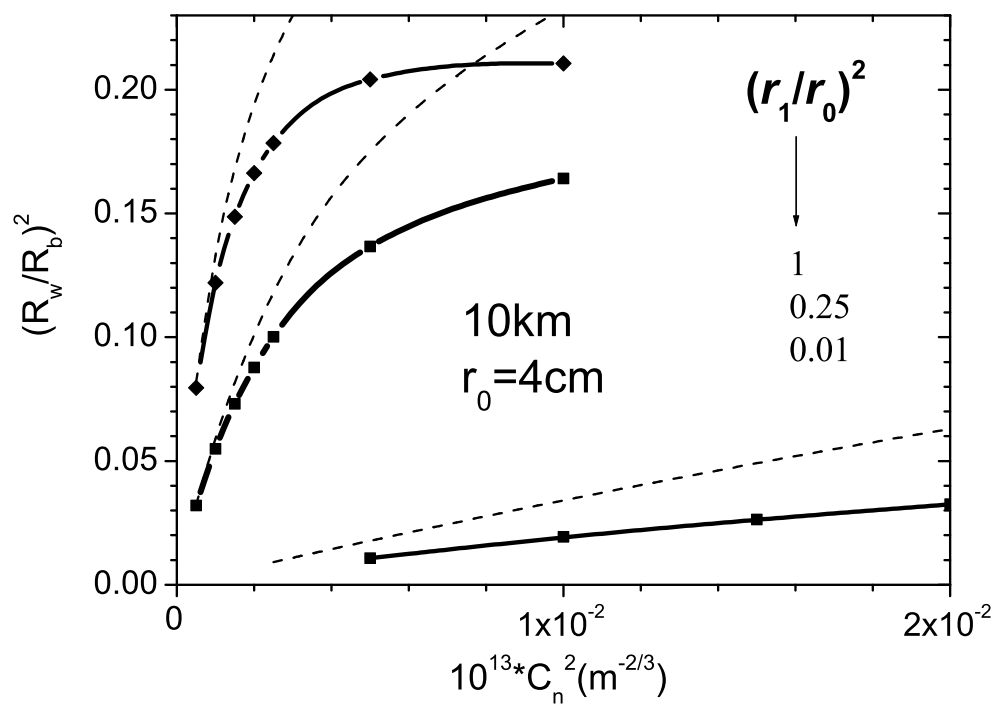

Figure 2: Dependence of beam wandering on the turbulence strength for small values of $C_{n}^{2}$. Dashed lines show the dependence given by Eq. (12), solid lines with symbols show the results of more general theory.

Fig. 4 illustrates how the two approaches correspond to one another at small values of $C_{n}^{2}$. Again we see a good agreement of both theories in this range of $C_{n}^{2}$.

Fig. 5 illustrates the dependence of the ratio $R_{w}^{2} / R_{b}^{2}$ on the turbulence strength for small values of the aperture radius $\left(r_{0}=1 \mathrm{~cm}\right)$. There is a significant decrease of the wandering effect in this case. This is because a small value of $r_{0}$ (and automatically $r_{1}$ ) results in considerable diffraction broadening of the beam for such a long propagation path $(5 \mathrm{~km})$. That is why the influence of turbulence on the beam parameters becomes competitive at greater values of $C_{n}^{2}$ and, in correspondence with the latter, the maxima of both curves are displaced to the right as compared with Fig. 3. Also, the effect of partial coherence is more pronounced for smaller initial radius of the beam. This can be seen by comparing Figs. 3 and 5 .

The results presented in Figs. 1-5 require additional comments. Our analysis proceeds from Eq. (2) where the evolution of the distribution function is based on the kinetic equation (4). By definition this function is quadratic 


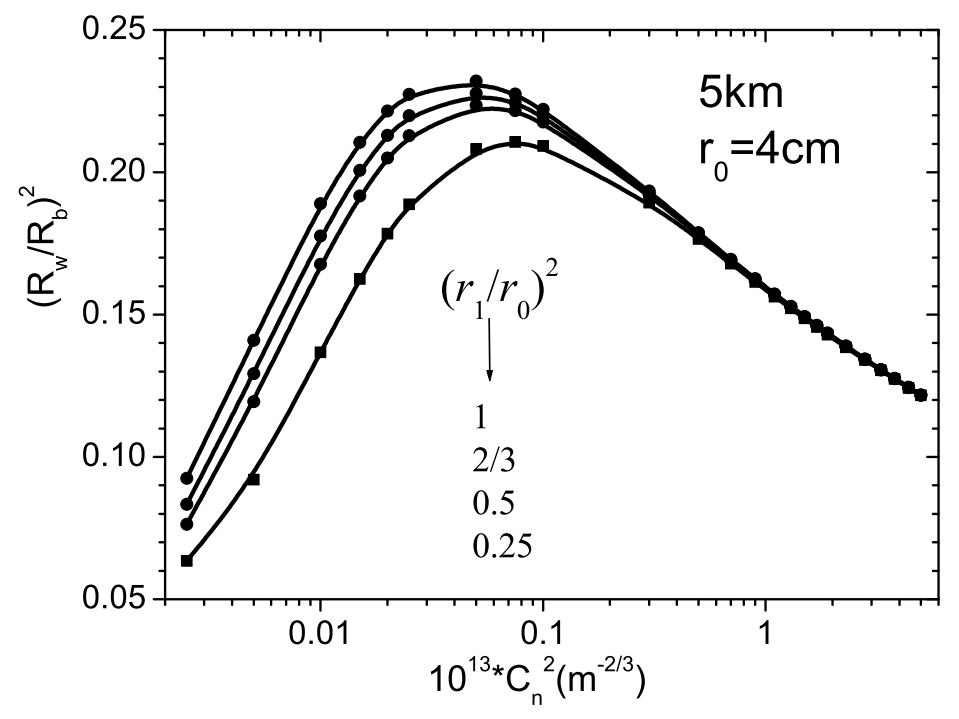

Figure 3: The same as in Fig. 1 but for $z=5 \mathrm{~km}$.

in the field amplitudes describing the intensity of the irradiance. Not all momenta $\mathbf{q}_{\perp}$ and wave-vectors $\mathbf{k}_{\perp}$ contribute to the observable intensity. One can easily see that initially in the absence of a phase screen, the characteristic values of both $q_{\perp}$ and $k_{\perp}$ are given by $r_{0}^{-1}$. In the presence of phase screen, the characteristic value of $q_{\perp}$ is of the order of $r_{1}^{-1}$ that determines the divergence of the beam and its broadening (diffraction broadening). It follows from geometric consideration that the diffraction broadening is of the order of $z^{2} q_{0}^{-2} r_{1}^{-2}$. This is almost the same value as given by Eq. (18). Therefore the characteristic value of $k_{\perp}$ decreases with distance as $\left(r_{0}^{2} / 4+z^{2} r_{1}^{-2} q_{0}^{-2}\right)^{-1 / 2}$. Also, the momentum $\mathbf{q}_{\perp}$ of the moving particle varies with distance due to scattering on atmosphere inhomogeneities. The additional momentum acquired in this way, $\Delta q_{\perp}$, can be estimated from its mean square value as in the case of a Brownian particle moving in $\mathbf{q}_{\perp}$-space and being affected by a random force $\mathbf{F}_{\perp}$ during the time $t=z / c$. Thus we have

$$
\left\langle\Delta q_{\perp}^{2}\right\rangle \sim\left\langle F_{\perp}^{2}\right\rangle t \sim C_{n}^{2} z
$$

As a result, the beam becomes more divergent and additional broadening 


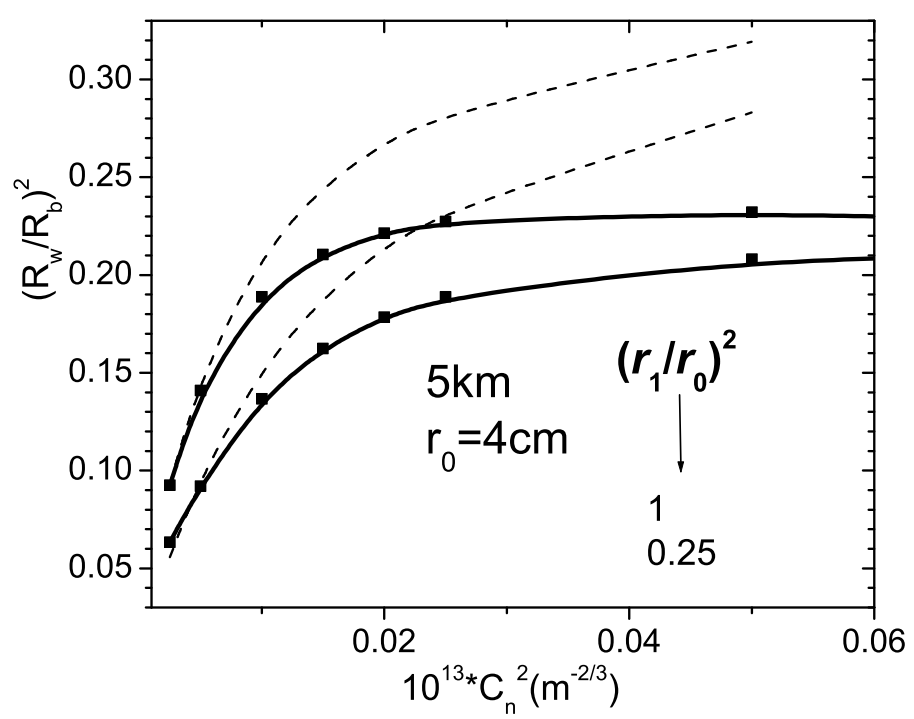

Figure 4: The same as in Fig. 2 but for $z=5 \mathrm{~km}$.

due to the turbulence, $\Delta R_{b}^{2}$, can be estimated as

$$
\Delta R_{b}^{2} \approx\left\langle\left[\frac{1}{q_{0}} \int_{0}^{z} d z^{\prime} \Delta q_{\perp}\left(z^{\prime}\right)\right]^{2}\right\rangle \sim C_{n}^{2} z^{3},
$$

. This again agrees with Eq. (18), where $\Delta R_{b}^{2}=2 \cdot 23 l_{0}^{-1 / 3} C_{n}^{2} z^{3}$.

When

$$
\Delta R_{b}^{2}>>\frac{r_{0}^{2}}{2}+\frac{2 z^{2}}{q_{0}^{2}} r_{1}^{-2},
$$

one can say that the beam size is determined almost entirely by the effects of the turbulence. In this case the characteristic values of $k_{\perp}$ are of the order of $\left(\Delta R_{b}\right)^{-1}$ and decrease with the increasing turbulence as $C_{n}^{-1} z^{-3 / 2}$. Also the characteristic value of $q_{\perp}$ becomes of the order of $\Delta q_{\perp}$ which is much greater than its initial value $r_{0}^{-1}$ (or $r_{1}^{-1}$ ). The last point can be seen directly from Eq. (19) when we represent the turbulence broadening as

$$
\Delta R_{b}^{2} \sim \frac{z^{2}}{q_{0}^{2}} \Delta q_{\perp}^{2} .
$$

The condition $\Delta q_{\perp}>>r_{0}^{-1}, r_{1}^{-1}$ means a considerable randomization of the radiation field. The waves acquire properties of Gaussian statistics that is 


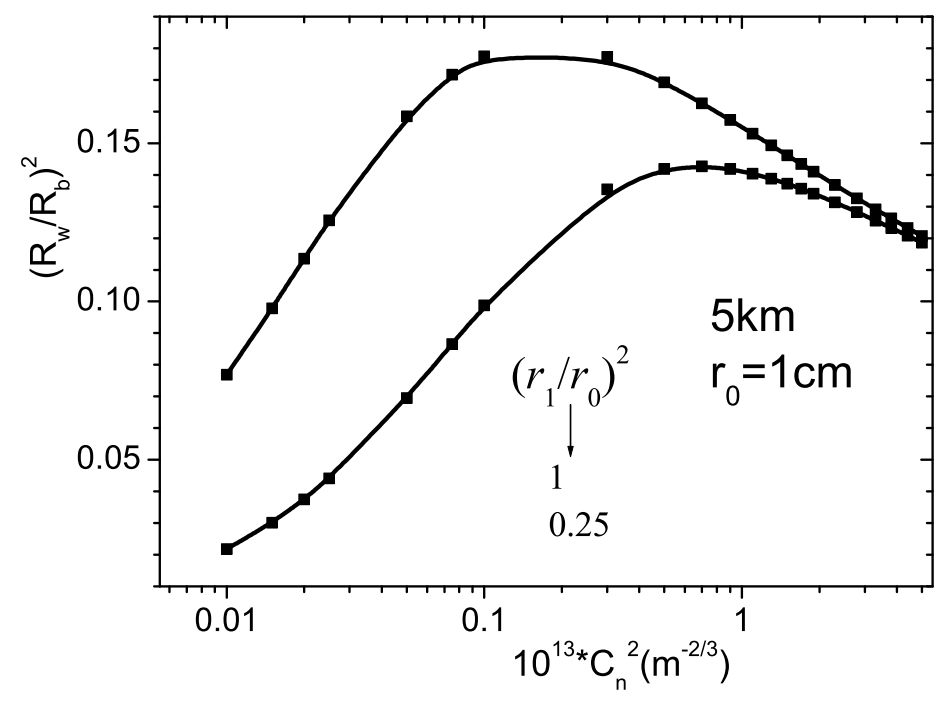

Figure 5: The same as in Fig. 3 but for $r_{0}=1 \mathrm{~cm}$.

very important when calculating beam wander variance. In contrast to calculations of the beam radius which is determined by correlations of only two waves, the beam wandering effect is determined by four-wave correlations (or by pair correlation function of the intensity $\langle I I\rangle)$. The results presented in Figs. 1-5 were obtained explicitly assuming the dominating contribution to the average

$$
\left\langle I(\mathbf{r}) I\left(\mathbf{r}^{\prime}\right)\right\rangle \sim \sum_{\mathbf{q}, \mathbf{k}} \sum_{\mathbf{q}^{\prime}, \mathbf{k}^{\prime}} e^{-i\left(\mathbf{k r}+\mathbf{k}^{\prime} \mathbf{r}^{\prime}\right)}\left\langle b_{\mathbf{q}+\mathbf{k} / 2}^{+} b_{\mathbf{q}-\mathbf{k} / 2} b_{\mathbf{q}^{\prime}+\mathbf{k}^{\prime} / 2}^{+} b_{\mathbf{q}^{\prime}-\mathbf{k}^{\prime} / 2}\right\rangle
$$

to be from small regions of $\mathbf{k}$ and $\mathbf{k}^{\prime}$ as explained above. (To simplify the notation, we omit the indices $(\perp)$ in all variables.) In this way strong correlations of pairs of waves $b_{\mathbf{q}+\mathbf{k} / 2}^{+}, b_{\mathbf{q}-\mathbf{k} / 2}$ and $b_{\mathbf{q}^{\prime}+\mathbf{k}^{\prime} / 2}^{+}, b_{\mathbf{q}^{\prime}-\mathbf{k}^{\prime} / 2}$ were taken into account. At the same time it is evident that there is another region of wave vectors, i.e.

$$
\left|\mathbf{q}+\mathbf{k} / 2-\mathbf{q}^{\prime}+\mathbf{k}^{\prime} / 2\right|,\left|\mathbf{q}-\mathbf{k} / 2-\mathbf{q}^{\prime}-\mathbf{k}^{\prime} / 2\right| \sim \Delta R_{b}^{-1}
$$

where pair correlations of waves may be also essential. The waves from different pairs, shown above, may correlate in this region. Conventionally, we will 
refer to this type of correlations as cross-correlation. In the case of strong turbulence, the contribution of cross-correlations is not small, thus providing saturation of fluctuations at high level. (See, for example, [6].) The two regions of wave vectors are well separated from one another and possible overlapping in the course of summing over wave vectors is not important in the case of strong turbulence [13. When the turbulence effect becomes weaker, these regions approach each other, and in the limit of small turbulence they unite into a single region. In this case the beam wander is determined by the asymptotically exact solution (12).

For strong turbulence, the contribution of cross correlations to the beam wandering, $\left\langle R_{w}^{2}\right\rangle_{\text {cross }}$, can be obtained as done in previous calculations. It is given by

$$
\left\langle R_{w}^{2}\right\rangle_{\text {cross }}=\frac{8}{3} \frac{r_{1}^{2}}{r_{0}^{2}} \frac{z^{2}}{q_{0}^{2} \Delta R_{b}^{2}} .
$$

Let us compare the value $\left\langle R_{w}^{2}\right\rangle_{\text {cross }}$ to $\left\langle R_{w}^{2}\right\rangle$, shown in Figs. 1-5. First of all, consider those $C_{n}^{2}$ which correspond to the maxima in the curves plotted in Figs. 1,3,5. For the case $r_{0}=r_{1}$ we see that $\Delta R_{b}^{2}>>\frac{r_{0}^{2}}{2}, \frac{2 z^{2}}{q_{0}^{2} r_{0}^{2}}$ in all cases. This means that these maxima are in the range of strong turbulence, and Eq. (20) is applicable here. The values obtained from Eq. (20) consist of only $7 \%, 5 \%, 0.4 \%$ of the corresponding data in Figs. 1,3,5, respectively. Moreover, if one moves towards greater values of $C_{n}^{2}$, the contribution of cross-correlations will become of smaller because of increasing of $\Delta R_{b}^{2}$. A similar situation occurs when $r_{1}$ becomes less than $r_{0}$.

On the other hand, our solutions with cross-correlations neglected almost coincide with those given by weak-turbulence theory when $C_{n}^{2} \rightarrow 0$. (See Figs. 2 and 3.) This assures us that Figs. 1,3,5 represent reasonable solutions for the specific set of parameters used there (and close to those) for any values of the turbulence strength, $C_{n}^{2}$.

\section{Conclusion}

We have applied the method of photon distribution function [6] to describe beam wander in turbulent atmosphere. In the limit of weak turbulence and in the absence of artificial random phase modulation, it becomes possible to obtain an analytical expression for the wandering radius, which coincides with the one known in the literature. Also, by bringing together analytical 
and numerical calculations, we have succeeded in obtaining the wandering radius in the range of strong turbulence. The general conclusion of the actual studies is that the variation of the initial spatial and temporal coherence provides significant positive (from the viewpoint of practical applications of laser beams) influence on the character of the intensity fluctuations. Namely, the relative value of the wandering radius can be considerably reduced. Moreover this reduction takes place just in the range of the most pronounced wandering effect. (See Figs. 1,3,5.) At the same time, the effect of partial coherence vanishes for very strong turbulence. This is in contrast to the behavior of the scintillation index, which in this case can be significantly suppressed by decreasing the initial coherence of the light. (See, for example, Refs. [4 and [6].) But this suppression is not very important because of small wandering effect in this case.

\section{Acknowledgment}

We thank B.M. Chernobrod, G.D. Doolen, and P.W. Milonni for discussions. This work was carried out under the auspices of the National Nuclear Security Administration of the U.S. Department of Energy at Los Alamos National Laboratory under Contract No. DE-AC52-06NA25396.

\section{References}

[1] G. Gbur and E. Wolf, J. Opt. Soc. Am. A, 19, 1592 (2002).

[2] J.C. Ricklin and F.M. Davidson, J. Opt. Soc. Am. A 191794 (2002).

[3] A. Dogariu and S. Amarande, Opt. Lett. 2810 (2003).

[4] V.A. Banakh, V.M. Buldakov, and V.L. Mironov, Opt. Spectrosk., 54, 1054 (1983).

[5] O. Korotkova, L.C. Andrews, and R.L. Phillips, Opt. Eng., 43, 330 (2004).

[6] G.P. Berman and A.A. Chumak, Phys. Rev. A74, 013805 (2006). 
[7] G.P. Berman, A.R. Bishop, B.M. Chernobrod, D.C. Nguyen, and V.N. Gorshkov, http://arxiv.org/ftp/physics/papers/0702/0702038.pdf, pp. $1-16$.

[8] G.P. Berman and A.A. Chumak, arXiv:quant-ph/0702238 v1 26 Feb 2007.

[9] R.J. Cook, J. Opt. Soc. Amer., 65, 942 (1975).

[10] R.L. Fante, Proc. IEEE, 63, 1669 (1975).

[11] R.L. Fante, Proc. IEEE, 68, 1424 (1980).

[12] A. Belmonte, Appl.Opt., 39, 5428 (2000).

[13] Our explanations are very similar to Dashen's observations who studied the problem of the intensity fluctuations using a path integral method. He noticed that for strong turbulence, there are two important regions of path space where the quadruple path integral factors into a product of two double path integrals, each of which is close to the integral encounted in the calculations of the field intensity. R. Dashen, J. Math. Phys., 20, 894 (1979). 